\title{
Chelators influenced synthesis of chitosan-carboxymethyl cellulose microparticles for controlled drug delivery
}

\author{
Antony V. Samrot ${ }^{1} \cdot$ Akanksha $^{1} \cdot$ Tatipamula Jahnavi $^{1} \cdot$ S. Padmanaban ${ }^{1}$. \\ Sheryl-Ann Philip ${ }^{1} \cdot$ Ujjala Burman $^{1} \cdot$ Arul Maximus Rabel $^{1}$
}

Received: 31 May 2016/Accepted: 22 June 2016/Published online: 1 July 2016

(c) The Author(s) 2016. This article is published with open access at Springerlink.com

\begin{abstract}
In this study, polyphenolic curcumin is entrapped within microcomposites made of biopolymers chitosan (CS) and carboxymethyl cellulose (CMC) formulated by ionic gelation method. Here, different concentrations of two chelating agents, barium chloride and sodium tripolyphosphate, are used to make microcomposites. Thus, the synthesized microparticles were characterized by FTIR, and their surface morphology was studied by SEM. Drug encapsulation efficiency and the drug release kinetics of CS-CMC composites are also studied. The produced microcomposites were used to study antibacterial activity in vitro.
\end{abstract}

Keywords Chitosan (CS) - Carboxymethyl cellulose $(\mathrm{CMC}) \cdot$ Sodium tripolyphosphate (TPP) · Barium chloride

\section{Introduction}

The main aim of any drug therapy against any disease is to release the desired therapeutics at the targeted site and also to maintain the concentration for the entire duration of treatment (Mitra and Dey 2011). Microparticles are small particles with the diameter ranging between 1 and $1000 \mu \mathrm{m}$, made of either natural or synthetic materials. Microparticles used for drug delivery should be biocompatible and biodegradable. The design of controlled release by microparticles depends on the nature of the

Antony V. Samrot

antonysamrot@gmail.com

1 Department of Biotechnology, Sathyabama University, Jeppiaar Nagar, Sholinganallur, Chennai, Tamil Nadu 600 119, India polymer, method adapted and various other factors which affect its surface properties (Edwards et al. 1997; Andrianov and Payne 1998; Zimmer and Kreuter 1995; Kurita 1986). Natural biopolymers, like chitosan (CS), carboxymethyl cellulose (CMC), polyethylene glycol (PEG), poly(lactic acid) (PLA), polyhydroxyalkanoates (PHA) etc., can be used for drug delivery (Wang et al. 2011; Yue et al. 2013). Among these biopolymers, chitosan made of polymer of glucosamine and $N$-acetyl glucosamine is absolutely biocompatible and biodegradable, which has also got flexibility in reacting with polyanions to shape edifices and gels (Shahidi and Synowiecki 1991; Sanford 1989). Chitosan microspheres/nanoparticles can be prepared by various methods like precipitation, complex coacervation, modified emulsification, ionotropic gelation, glutaraldehyde cross-linking, thermal cross-linking etc. (Kas 1997; Yao et al. 1995). CMC has got high viscosity, good solubility and high chemical stability. CMC is also an excellent mucoadhesive, safe, hydrophilic, biocompatible and biodegradable (Smart et al. 1994). CMC is an anionic polysaccharide, which has been proven to be nontoxic as well as biodegradable. Moreover, CMC can easily form a microparticle or nanoparticle and can be used for drug delivery (Chandy and Sharma 1990; Cerchiara et al. 2016; Bigucci et al. 2015; Garcia et al. 2015; Lu et al. 2010). In acid medium, the weak base CS can react with an anionic polymer, like TPP and CMC, to form microparticle (Chandy and Sharma 1990; Takishima et al. 2002). CUR is a curcuminoid isolated from the rhizome of Curcuma longa L. (Zingiberaceae family) with the common name of turmeric (Ammon and Wahl 1991). CUR is well known for its various bioactivities such as antioxidant, antimicrobial, anticarcinogenic etc. (Wright et al. 2013; Niamsa and Sittiwet 2009). The drawback of CUR is that it has poor solubility in aqueous 
media, low bioavailabilty, poor circulation time and degrades easily under physiological conditions (Jisha et al. 2015). CUR loaded-myristic acid microemulsion with $0.86 \mu \mathrm{g} \mathrm{ml}^{-1}$ of CUR is suitable for skin consumption, which inhibits $50 \%$ of the S. epidermidis growth, one of the nosocomial infectious agents (Liu and Huang 2012). Nanoformulation of CUR with synthetic polymer and liposomes has been effectively carried out to increase the solubility and degradability of hydrophobic CUR drugs (Yu and Huang 2012). In this study, barium chloride and TPP are anions which bring the amine gathering of CS and cross-links CMC. Here, CS microparticles epitomize the CUR drug, and the synthesized microparticles are characterized using various instrumental methods like FTIR, SEM, encapsulation efficiency, drug release kinetics and in vitro controlled release studies against Pseudomonas aeruginosa.

\section{Materials and methods}

\section{Materials}

All the chemicals and reagents used in this study were purchased of analytical grade. The synthesis of microcarriers has been completed with the following chemicals: barium chloride, chitosan extrapure $\left(\mathrm{C}_{6} \mathrm{H}_{11} \mathrm{NO}_{4}\right)$, curcumin and agar powder were obtained from Sisco Research Laboratories Pvt. Ltd; acetic acid was purchased from Qualigens Fine Chemicals; sodium tripolyphosphate (anhydrous) was purchased from Loba Chemie; carboxymethyl cellulose was purchased from Micro Fine Chemicals, India; ethyl alcohol AR was obtained from Changshu Yangyuan Chemicals; Milli-Q water was used for all the chemical preparations; and curcumin was used as the anticancer drug to be loaded in the microcarriers for the study.

\section{Preparation of microcarriers using TPP}

$0.4 \mathrm{~g}$ of CS was dissolved in $100 \mathrm{ml}$ acetic acid $(0.1 \mathrm{~N})$. $0.2,0.4$ and $0.6 \%$ TPP were prepared in $50 \mathrm{ml}$ deionized water (Calvo et al. 1997). TPP solution was added dropwise to CS solution at room temperature. $50 \mathrm{ml}$ of $0.4 \% \mathrm{CMC}$ was prepared in deionized water and was added dropwise to CS chelated with TPP solution. The solution was left for $2 \mathrm{~h}$ and centrifuged at $5000 \mathrm{rpm}$ for $15 \mathrm{~min}$. The microcarriers prepared with $0.2,0.4$ and $0.6 \%$ TPP were labeled as CS-CMC-S1, CS-CMC-S2 and $\mathrm{CS}-\mathrm{CMC}-\mathrm{S} 3$, respectively. The solutions were lyophilized. Here, microcarriers are formed due to the interaction of oppositely charged cross-linking agent (Zhao et al. 2011).

\section{Preparation of microcarriers using barium chloride}

The above procedure was used to prepare microcarriers using barium chloride replacing TPP. The microcarriers prepared with $0.2,0.4$ and $0.6 \%$ barium chloride were labeled as CS-CMC-B1, CS-CMC-B2 and CS-CMC-B3, respectively. The solutions were lyophilized and used for further studies.

\section{Preparation of CUR-encapsulated polymeric microcarriers}

CUR was loaded into polymeric microcarriers following ionic gelation method. $0.05 \mathrm{~g}$ of CUR was dissolved in $50 \mathrm{ml}$ ethanol. This was added to $0.4 \mathrm{~g}$ CS dissolved in $100 \mathrm{ml}$ acetic acid $(0.1 \mathrm{~N}) .0 .2,0.4$, and $0.6 \%$ barium chloride solution was added dropwise to CS-CUR solution, and CMC solution was added to ionized CS-CUR-barium chloride solution and left at room temperature for $2 \mathrm{~h}$. The solutions were centrifuged at $5000 \mathrm{rpm}$ for $15 \mathrm{~min}$. The microcarriers prepared with $0.2,0.4$ and $0.6 \%$ barium chloride were labeled as CS-CUR-CMC-B1, CS-CURCMC-B2 and CS-CUR-CMC-B3, respectively. The above method was repeated for the synthesis of CURencapsulated microparticles. The microcarriers prepared with $0.2,0.4$ and $0.6 \%$ TPP were labeled as CS-CUR-CMC-S1, CS-CURCMC-S2 and CS-CUR-CMC-S3, respectively.

\section{Characterization of the microcomposites}

\section{Fourier transfer infrared spectroscopy (FTIR) analysis}

IR spectrum was recorded using IRAffinity-1s (Shimadzu, Japan) instrument. Drug-loaded and unloaded microcarrier pellets were tested with transmission mode scan in the spectral region of $4000-400 \mathrm{~cm}^{-1}$.

\section{Scanning electron microscope (SEM) analysis}

The microcarriers with and without CUR loaded were sprayed onto glass plate and sputter-coated with gold and examined under SEM (JEOL JSM-5610LV).

\section{Evaluation of encapsulation efficiency}

The microparticles were separated by centrifugation at $3000 \mathrm{rpm}$ for $15 \mathrm{~min}$, and drug encapsulation efficiency (EE) of the microparticles was evaluated by measuring the absorption of the supernatant liquid using UV spectrophotometer at $425 \mathrm{~nm}$ ( $\lambda_{\max }$ of CUR) (Parize et al. 2012; Mukerjee and Vishwanatha 2009; Benetton et al. 1998). The supernatant was collected at different intervals, three 

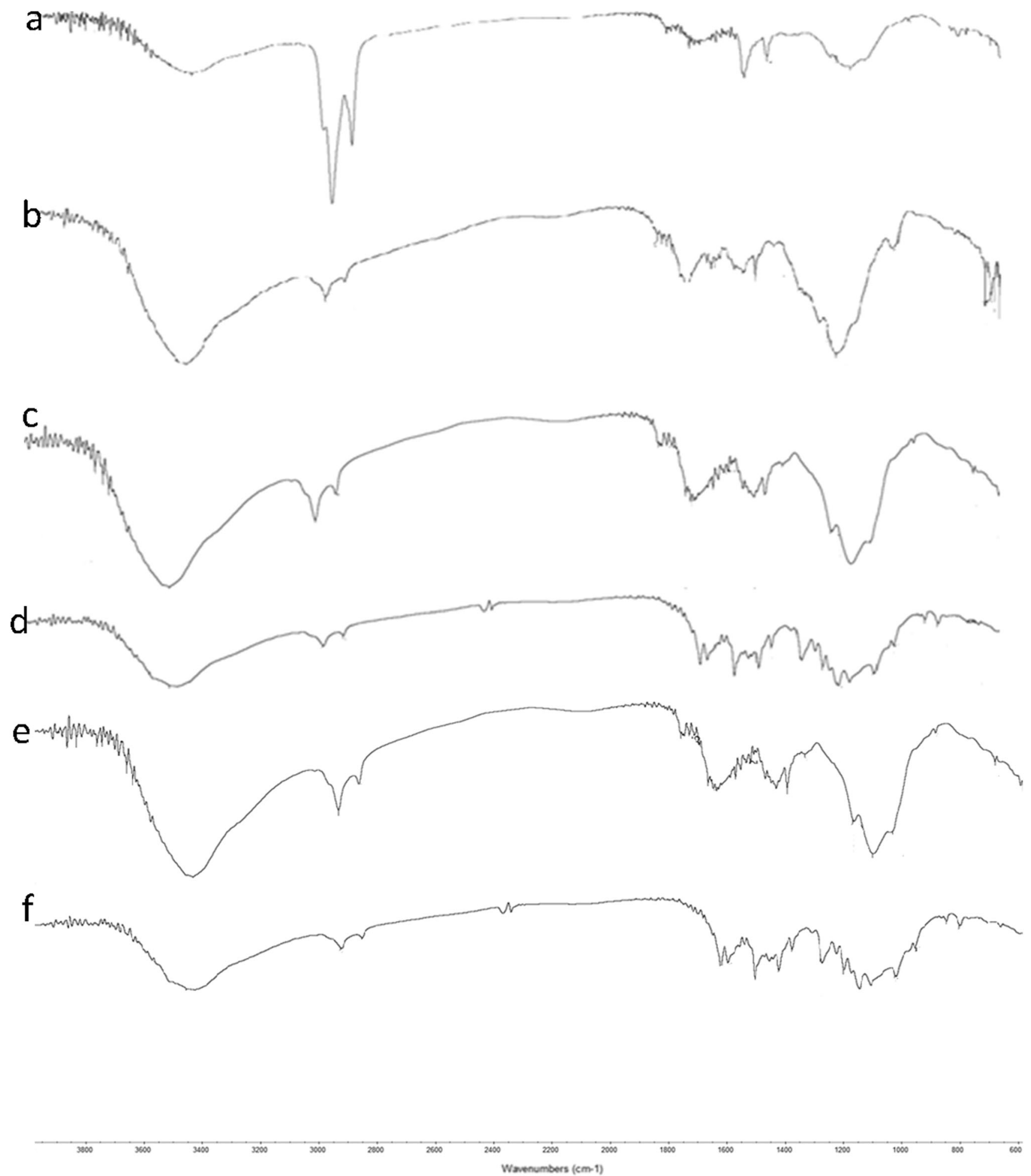

Fig. 1 FTIR analysis of CS microparticles chelated with $\mathrm{BaCl}_{2}$ $a$ chelated with $0.2 \% \mathrm{BaCl}_{2}$ and without drug (CS-CMC-B1), $b$ chelated with $0.2 \% \mathrm{BaCl}_{2}$ and loaded with drug (CS-CMC-CUR$\mathrm{B} 1$ ), $c$ chelated with $0.4 \% \mathrm{BaCl}_{2}$ and without drug (CS-CMC-B2), $d$ chelated with $0.4 \% \mathrm{BaCl}_{2}$ and loaded with drug (CS-CMC-CUR$\mathrm{B} 2), e$ chelated with $0.6 \% \mathrm{BaCl}_{2}$ and without drug (CS-CMC-B3), $f$ chelated with $0.6 \% \mathrm{BaCl}_{2}$ and loaded with drug (CS-CMC-CURB3) 
a
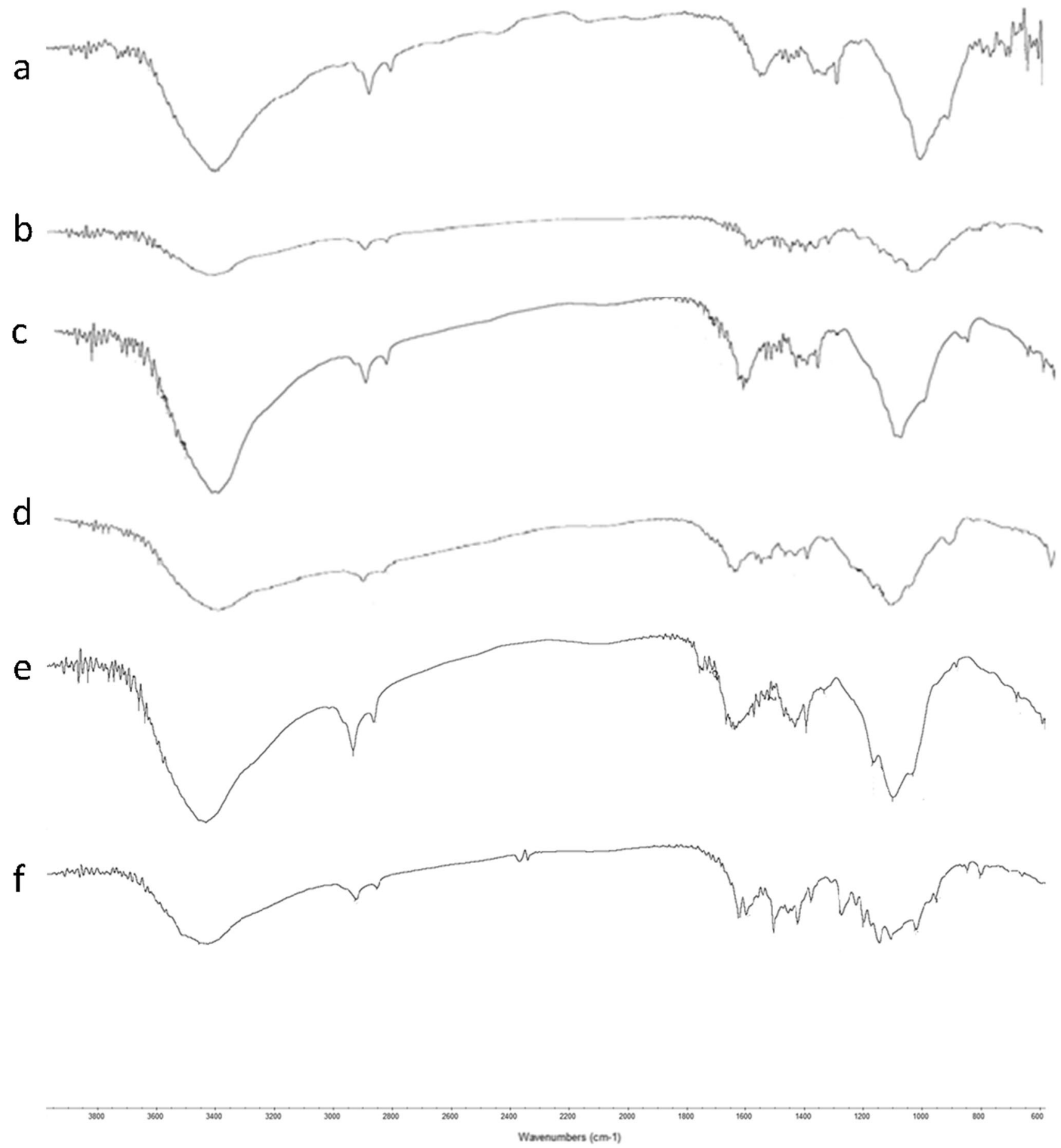

Fig. 2 FTIR analysis of CS microparticles chelated with TPP $a$ chelated with $0.2 \%$ TPP and without drug (CS-CMC-S1), $b$ chelated with $0.2 \%$ TPP and loaded with drug (CS-CMC-CUR$\mathrm{S} 1$ ), $c$ chelated with $0.4 \%$ TPP and without drug (CS-CMC-S2),

samples were taken, and the mean value and standard error were calculated. The graph was plotted by taking time in minutes on $x$ axis and absorbance in nm on $y$ axis from the values obtained. $d$ chelated with $0.4 \%$ TPP and loaded with drug (CS-CMC-CUR$\mathrm{S} 2), e$ chelated with $0.6 \%$ TPP and without drug (CS-CMC-S3), $f$ chelated with $0.6 \%$ TPP and loaded with drug (CS-CMC-CUR-S3)

\section{In vitro drug release kinetics}

The drug release kinetics for microcarriers was studied using dialysis membrane technique (Hua 2014) which is 
Fig. 3 SEM analysis of CS microparticles chelated with $\mathrm{BaCl}_{2}$ a chelated with $0.2 \%$ $\mathrm{BaCl}_{2}$ and without drug (CSCMC-B1), b chelated with $0.2 \% \mathrm{BaCl}_{2}$ and loaded with drug (CS-CMC-CUR-B1), c chelated with $0.4 \% \mathrm{BaCl}_{2}$ and without drug (CS-CMC-B2), d chelated with $0.4 \% \mathrm{BaCl}_{2}$ and loaded with drug (CSCMC-CUR-B2), e chelated with $0.6 \% \mathrm{BaCl}_{2}$ and without drug (CS-CMC-B3), f chelated with $0.6 \% \mathrm{BaCl}_{2}$ and loaded with drug (CS-CMC-CUR-B3)
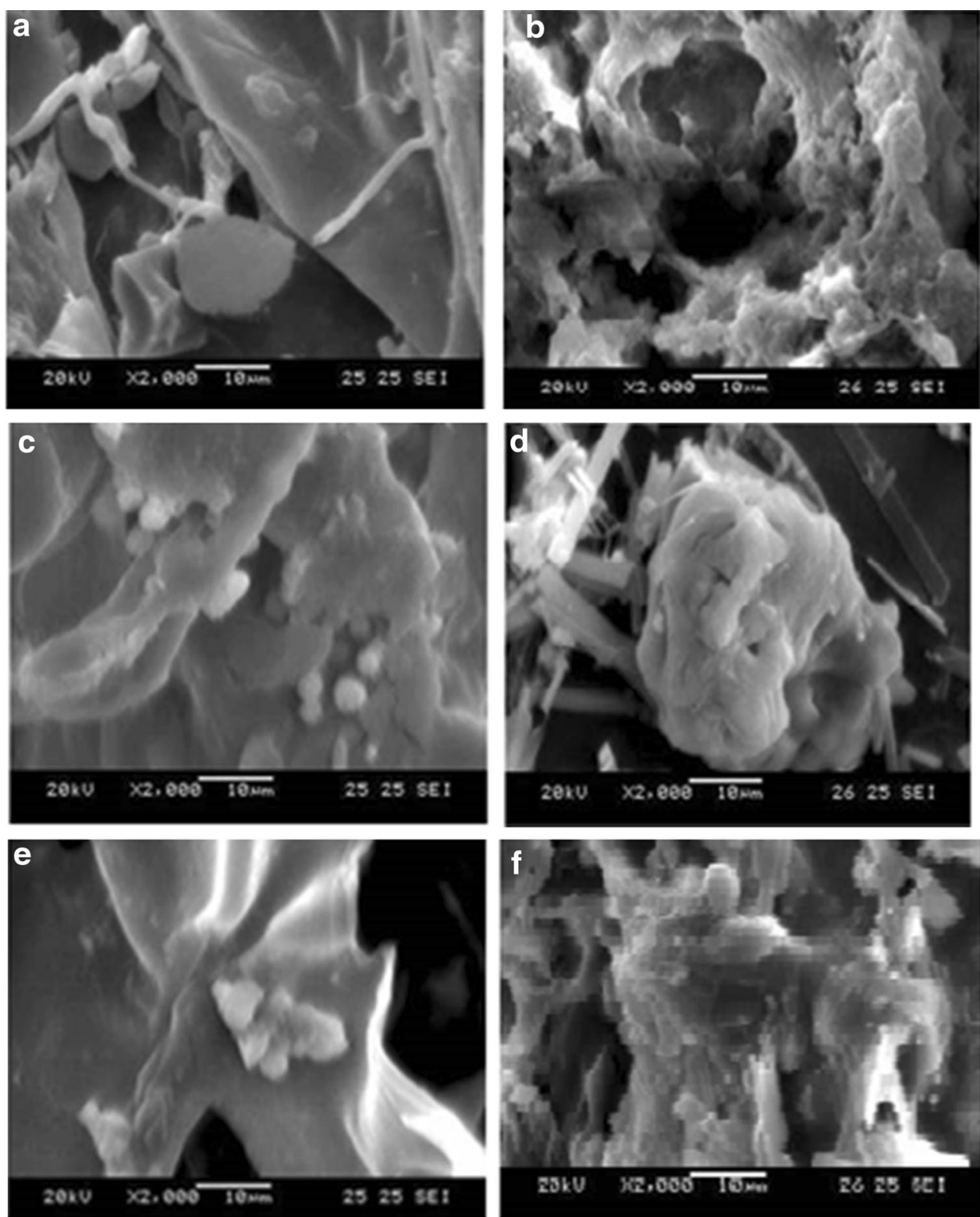

as follows: $10 \mathrm{mg}$ samples of (CS-CMC-B1, CS-CMCB2, CS-CMC-B3, CS-CUR-CMC-S1, CS-CUR-CMCS2 and CS-CUR-CMC-S3) were separately tied in six dialysis membranes (AV flat width-32.34 mm, AV diameter-21.5 mm, capacity approx-3.63 m cm $\mathrm{cm}^{-1}$ ). They were placed separately in $50 \mathrm{ml}$ of phosphate buffer solution ( $\mathrm{pH}$ 6.8) and left at room temperature. $1 \mathrm{ml}$ of the solution was collected at prescribed time intervals (i.e., 30, 60, 90, 120, 150, $180 \mathrm{~min}$ ) from the released medium. Three samples were taken, and the mean value and standard error were calculated. Absorbance was read using UV visible spectrophotometer (Systronics) at wavelength $425 \mathrm{~nm}$ ( $\lambda_{\max }$ of CUR). Graphs were plotted having time interval in $y$ axis and drug release absorbance in $x$ axis.

\section{In vitro controlled release studies}

\section{Antibacterial activity}

To know the bactericidal activity of microcomposites loaded with CUR, agar well diffusion method was performed (Dima et al. 2014; Buzia et al. 2015). In this method, MuellerHinton agar plates were swabbed all over with $P$. aeruginosa using a sterile swab. Wells were bored, and the different concentrations of microcomposites were added to the wells. After $48 \mathrm{~h}$, the zone of clearance was measured and recorded (Cruickshank 1962). CS microparticles were dissolved in different solvents like water, ethanol, phosphate buffer saline (PBS pH 6) and acetic acid at the concentration of $1 \mathrm{mg} \mathrm{ml}^{-1}$ and used to study the drug release kinetics.

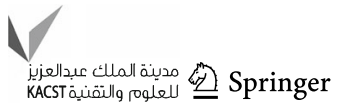


Fig. 4 SEM analysis of CS microparticles chelated with TPP a chelated with $0.2 \%$ TPP and without drug (CS-CMCS1), b chelated with $0.2 \%$ TPP and loaded with drug (CSCMC-CUR-S1), c chelated with $0.4 \%$ TPP and without drug (CS-CMC-S2), d chelated with $0.4 \%$ TPP and loaded with drug (CS-CMC-CUR-S2), e chelated with $0.6 \%$ TPP and without drug (CS-CMC-S3), f chelated with $0.6 \%$ TPP and loaded with drug (CS-CMCCUR-S3)
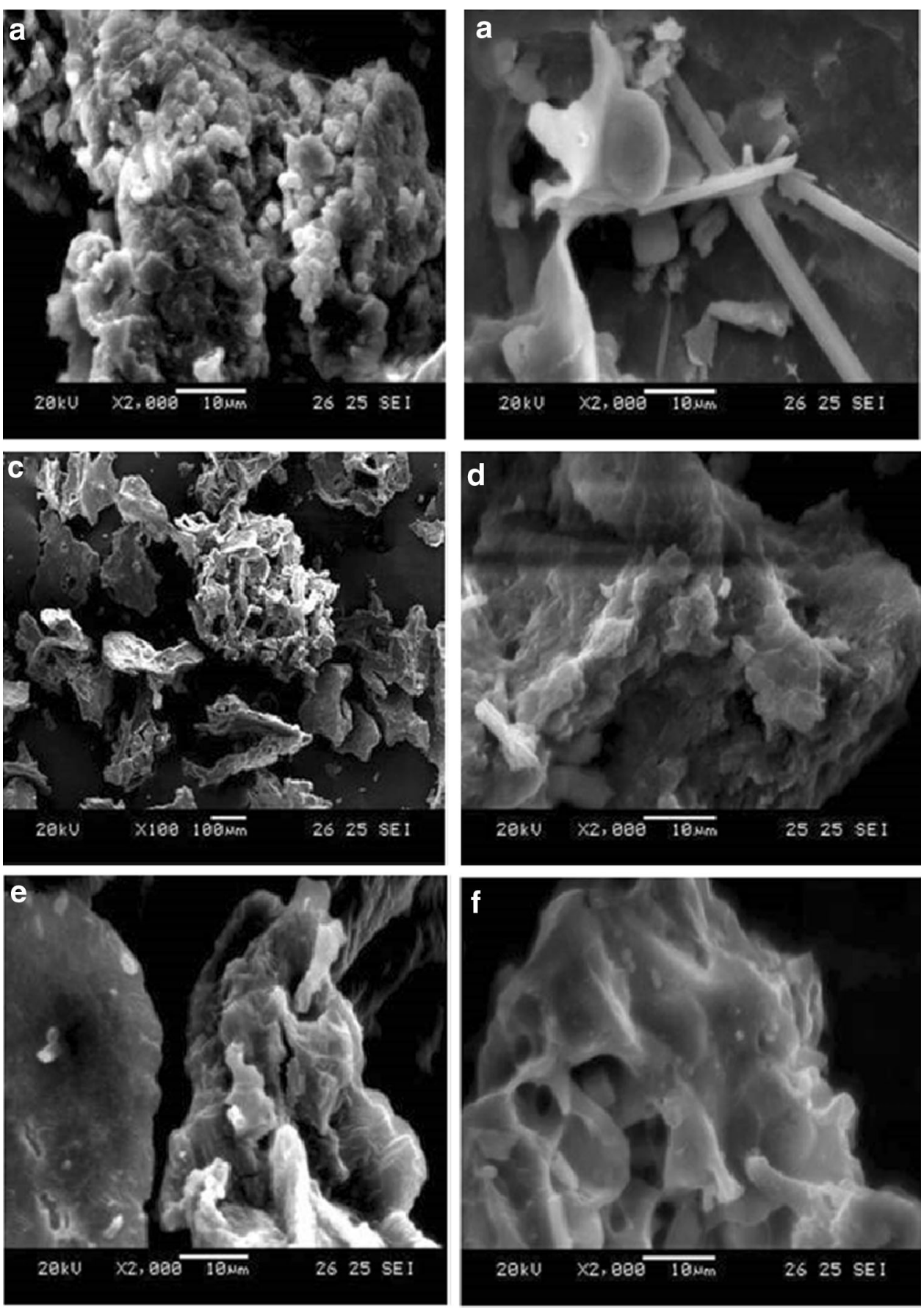

\section{Results and discussion}

\section{Fourier transfer infrared spectroscopy (FTIR) analysis}

FTIR analysis was carried out to confirm the presence of microcomposites and encapsulation of CUR. The FTIR spectra of CUR-loaded and unloaded microcomposites are shown in Figs. 1 and 2. The characteristic peaks for CSCMC-S1, CS-CMC-S2, CS-CMC-S3, CS-CMC-B1,
CS-CMC-B2 and CS-CMC-B3 were observed at $1633,1636,1636,1653,1636,1636 \mathrm{~cm}^{-1}$ which shows the presence of amide bonds representing the structure of $\mathrm{N}$ acetylglucosamine (Parize et al. 2012). The $\mathrm{C}-\mathrm{N}$ stretching vibration peaks for $\mathrm{CS}$ and $\mathrm{CMC}$ were observed at 1382 , 1384,1384, 1376, 1382 and $1383 \mathrm{~cm}^{-1}$ for all carrier samples CS-CMC-S1, CS-CMC-S2, CS-CMC-S3, CSCMC-B1, CS-CMC-B2 and CS-CMC-B3, respectively. FTIR of pure TPP showed characteristic bands at 1206-1215 (P-O stretching), 1135-1157 (symmetric and 
Fig. 5 Encapsulation efficiency of CUR on microparticles chelated with different concentration of $\mathrm{BaCl}_{2}$

Fig. 6 Encapsulation efficiency of CUR on microparticles chelated with different concentration of TPP
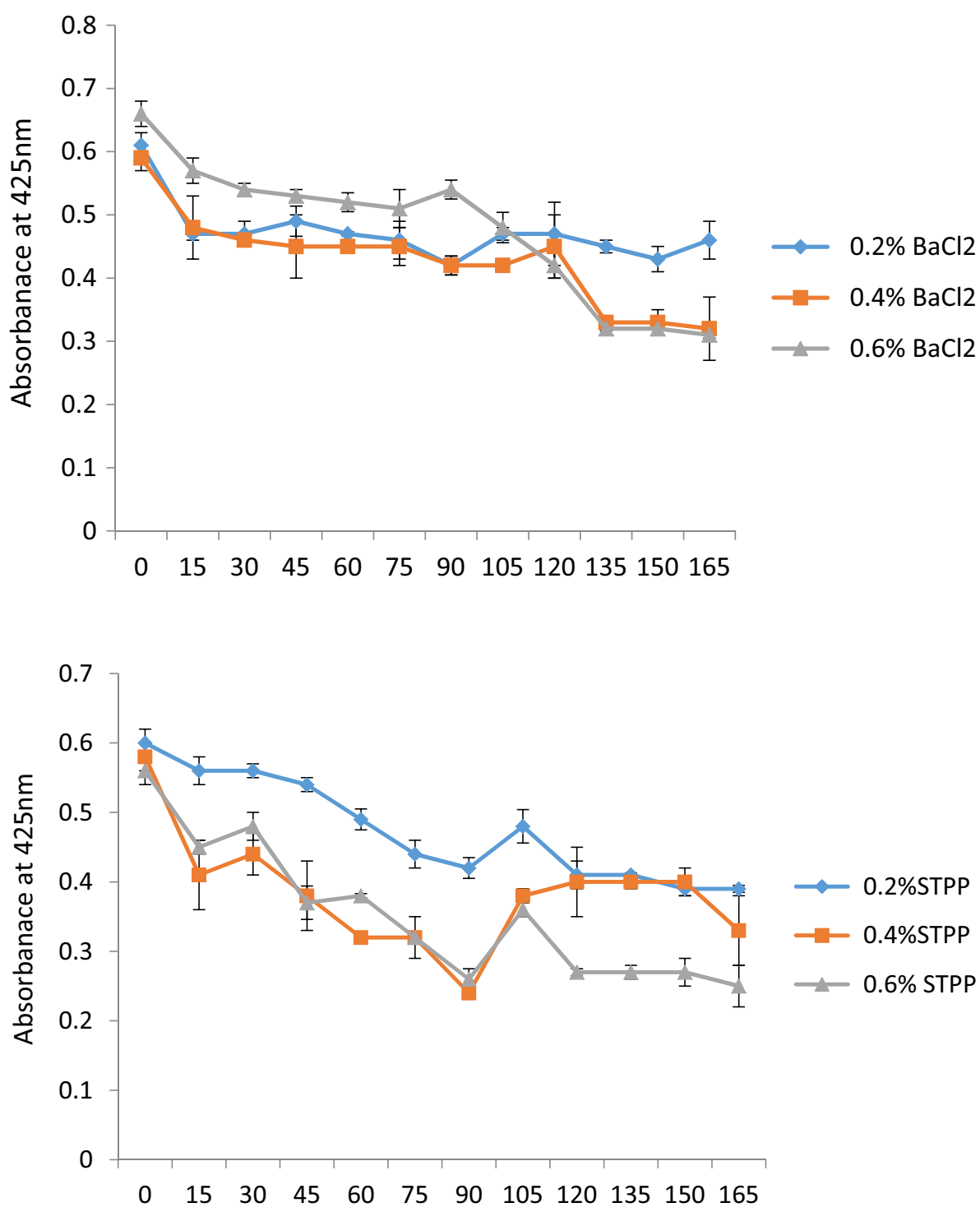

Fig. 7 UV-spectrophotometer analysis of microparticles chelated with barium chloride

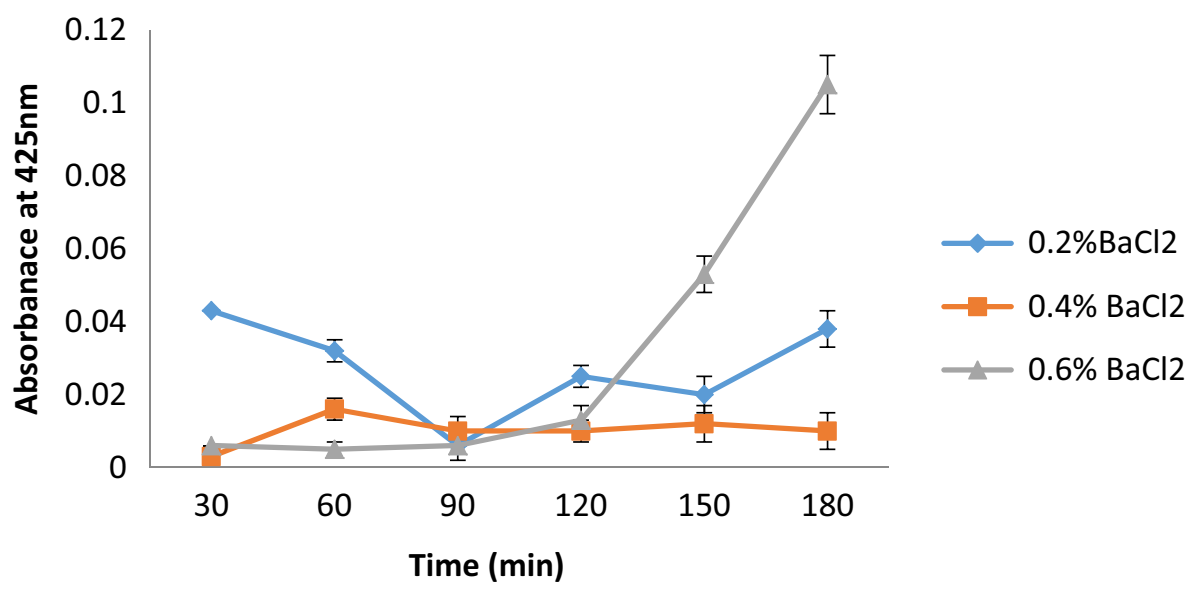


Fig. 8 UV-spectrophotometer analysis of microparticles chelated with TPP

Fig. 9 Antibacterial activity of CUR on Pseudomonas aeruginosa using water as solvent. a chelated with $0.2 \%$ $\mathrm{BaCl}_{2}$ and loaded with drug (CS-CMC-CUR-B1), b chelated with $0.4 \% \mathrm{BaCl}_{2}$ and loaded with drug (CSCMC-CUR-B2), c chelated with $0.6 \% \mathrm{BaCl}_{2}$ and loaded with drug (CS-CMC-CURB3). d Chelated with $0.2 \%$ TPP and loaded with drug (CSCMC-CUR-S1), e chelated with $0.4 \%$ TPP and loaded with drug (CS-CMC-CUR-S2), f chelated with $0.6 \%$ TPP and loaded with drug (CS-CMCCUR-S3)

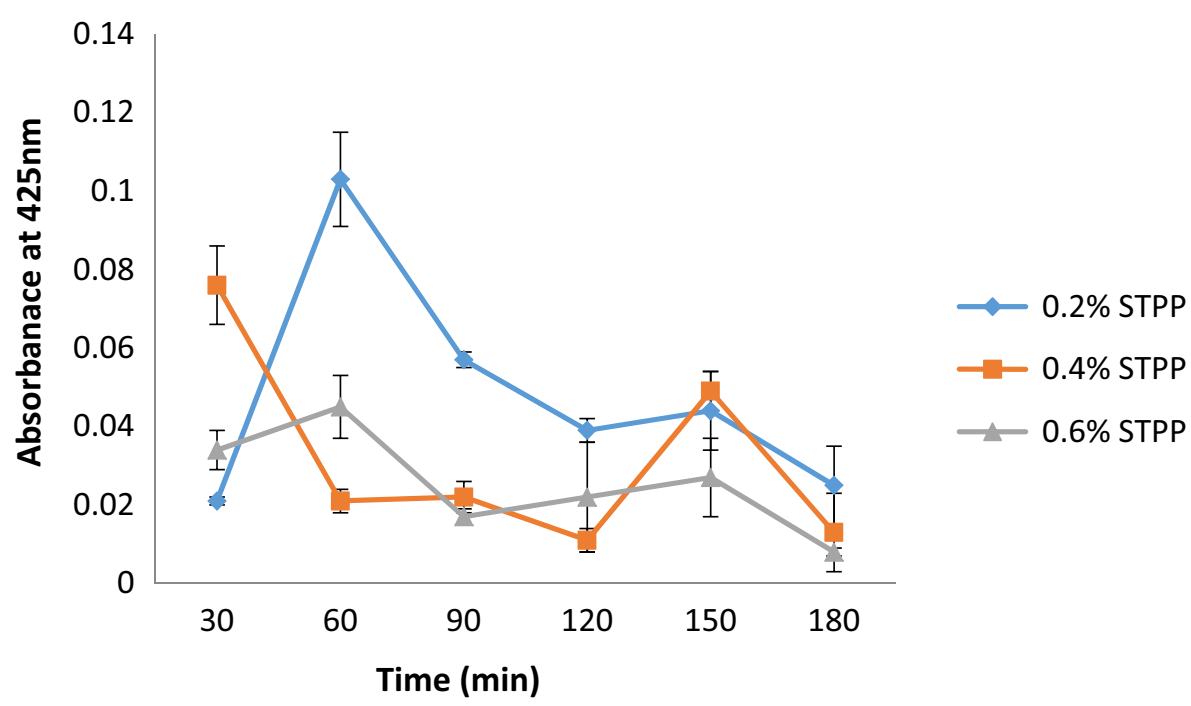

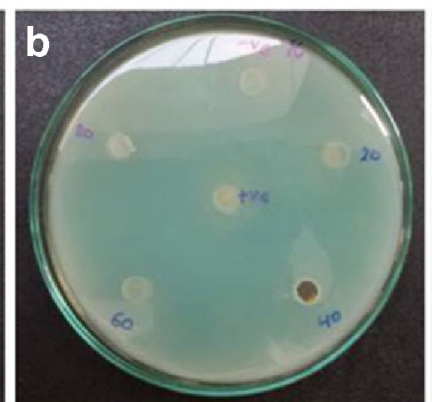
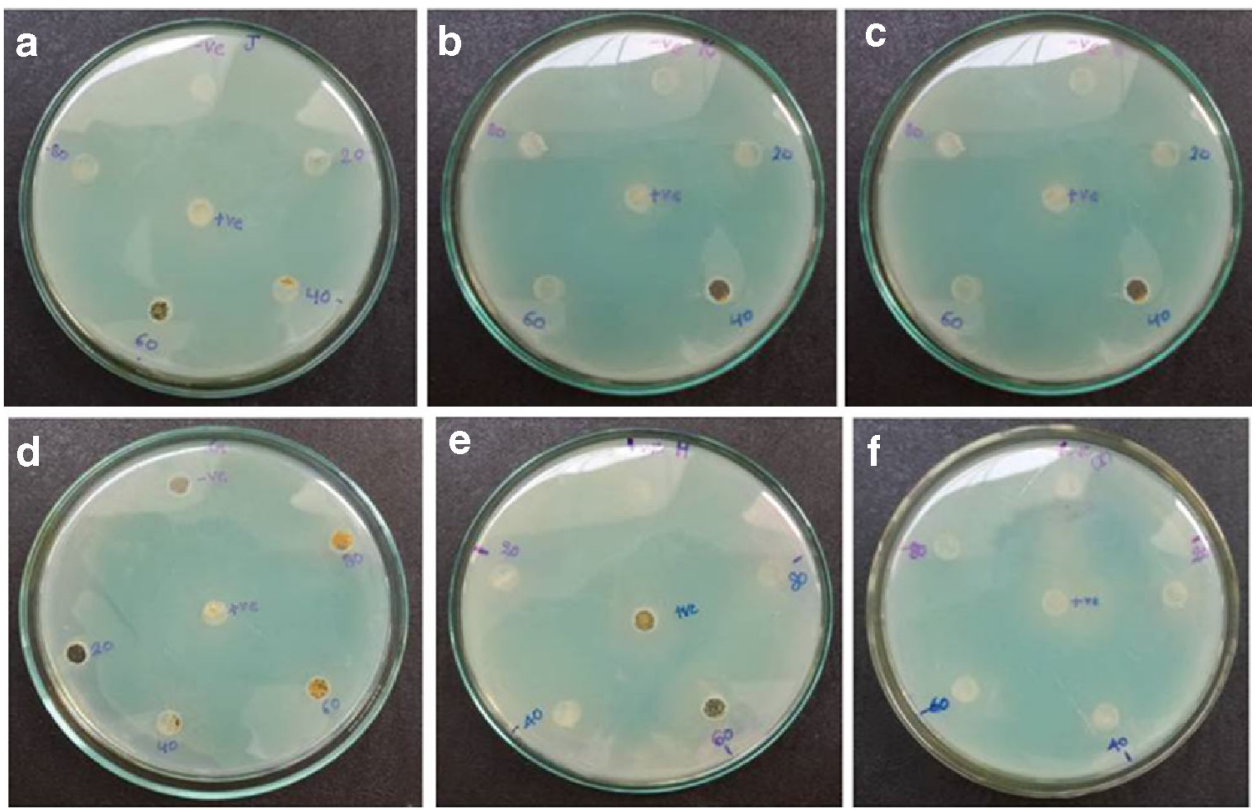

asymmetric stretching vibration of the $\mathrm{PO}_{2}$ groups), 1090-1115 (symmetric and asymmetric stretching vibration of the $\mathrm{PO}_{3}$ groups) and $880-895 \mathrm{~cm}^{-1}$ (P-O-P asymmetric stretching) (Mi et al. 1999; Martins et al. 2012) (Fig. 1a, c, e). The $\mathrm{C}=\mathrm{O}$ frequency was observed next to amide bond stretching which ranges from 1640 to $1650 \mathrm{~cm}^{-1}$. A broad trough was observed at 3448,3446 , $3446,3422,3446$ and $3422 \mathrm{~cm}^{-1}$ which shows the presence of either carbonyl or hydroxyl groups. The $\mathrm{C}-\mathrm{H}$ peak of CS was observed in all the drug-loaded and unloaded microcomposites at 2922, 2922, 2922, 2853, 2928, 2923, 2930, 2923, 2922, 2923, 2922, $2928 \mathrm{~cm}^{-1}$ (Figs. 1, 2). After CUR is loaded, the hydroxyl group peaks are 3421, $3421,3421,3422,3421$ and $3448 \mathrm{~cm}^{-1}$ for composites CS-CUR-CMC-S1, CS-CUR-CMC-S2, CS-CUR-
CMC-S3, CS-CUR-CMC-B1, CS-CUR-CMC-B2 and CS-CUR-CMC-B3 (Figs. 1b, d, f, 2b, d, f). The amine peak of the encapsulated polymer was observed at 1636 , $1636,1628,1636,1628$ and $1605 \mathrm{~cm}^{-1}$ for CS-CURCMC-S1, S2, S3, B1, B2 and B3, respectively (Figs. 1b, d, f, $2 \mathrm{~b}, \mathrm{~d}, \mathrm{f}) . \mathrm{C}=\mathrm{O}$ stretching was observed at 1653,1653 , 1654, 1653 and $1627 \mathrm{~cm}^{-1}$ for CUR microcomposites. The characteristic peaks of $\mathrm{OCH} 3$ for the presence of CUR were observed at $1153 \mathrm{~cm}^{-1}$ for all the samples except CS-CUR-CMC-B3 in which it was found to be at $1161 \mathrm{~cm}^{-1}$. C-N stretching was observed at 1384, 1383, $1384,1383,1383$ and $1386 \mathrm{~cm}^{-1}$ for microcomposites CSCUR-CMC-S1, CS-CUR-CMC-S2, CS-CUR-CMC-S3, CS-CUR-CMC-B1, CS-CUR-CMC-B2 and CS-CURCMC-B3, respectively (Figs. 1b, d, f, 2b, d, f). 
Fig. 10 Antibacterial activity of CUR on Pseudomonas aeruginosa using ethanol as solvent. a chelated with $0.2 \%$ $\mathrm{BaCl}_{2}$ and loaded with drug (CS-CMC-CUR-B1),

b chelated with $0.4 \% \mathrm{BaCl}_{2}$ and loaded with drug (CSCMC-CUR-B2), c chelated with $0.6 \% \mathrm{BaCl}_{2}$ and loaded with drug (CS-CMC-CUR-

B3). d chelated with $0.2 \%$ TPP and loaded with drug (CSCMC-CUR-S1), e chelated with $0.4 \%$ TPP and loaded with drug (CS-CMC-CUR-S2), f chelated with $0.6 \%$ TPP and loaded with drug (CS-CMCCUR-S3)

Fig. 11 Antibacterial activity of CUR on Pseudomonas aeruginosa using PBS as solvent. a chelated with $0.2 \%$ $\mathrm{BaCl}_{2}$ and loaded with drug (CS-CMC-CUR-B1), b chelated with $0.4 \% \mathrm{BaCl}_{2}$ and loaded with drug (CSCMC-CUR-B2), $\mathbf{c}$ chelated with $0.6 \% \mathrm{BaCl}_{2}$ and loaded with drug (CS-CMC-CURB3). d Chelated with $0.2 \%$ TPP and loaded with drug (CSCMC-CUR-S1), e chelated with $0.4 \%$ TPP and loaded with drug (CS-CMC-CUR-S2), f chelated with $0.6 \%$ TPP and loaded with drug (CS-CMCCUR-S3)
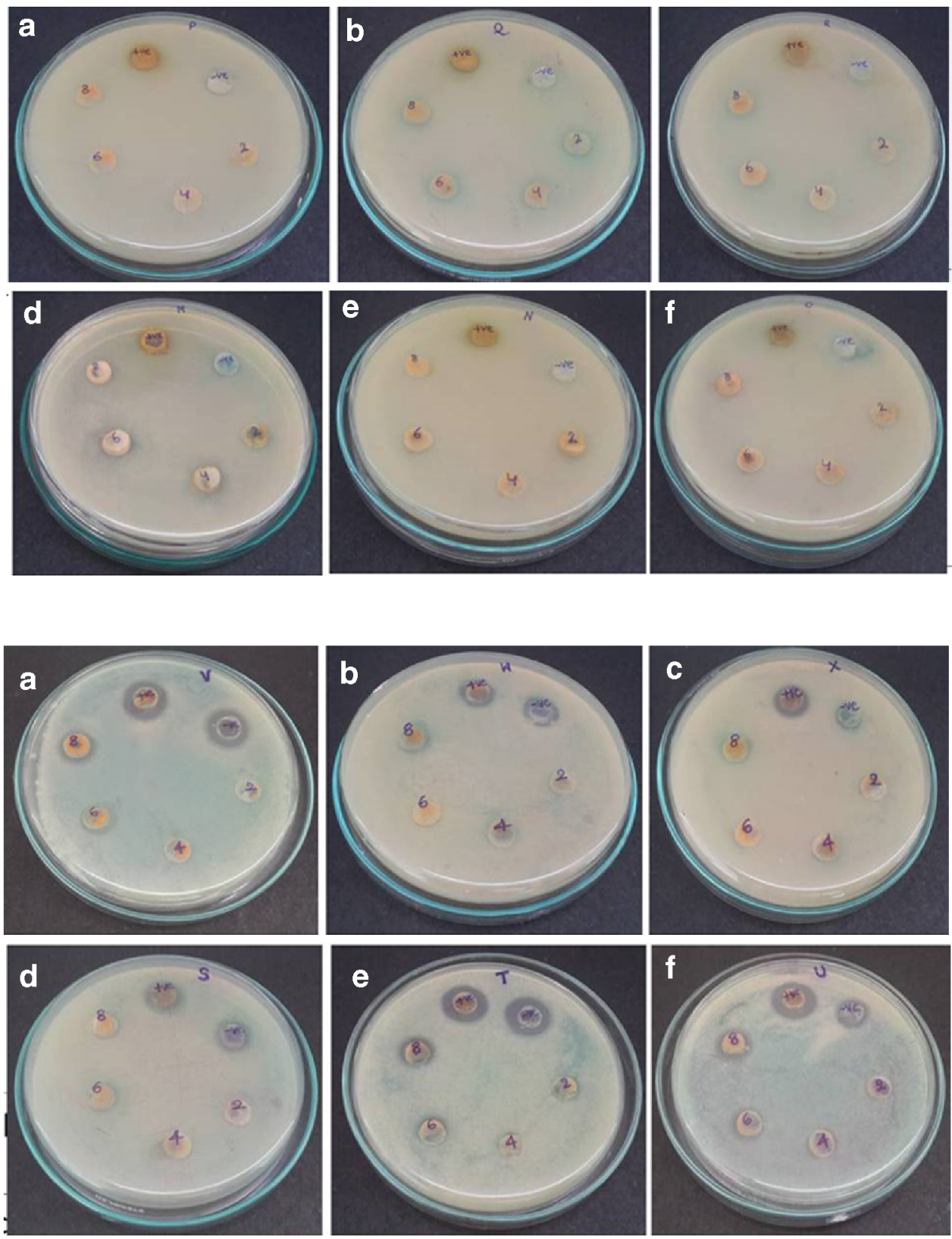

\section{Scanning electron microscopy (SEM) analysis}

SEM confirmed the surface morphology of microparticles chelated with barium chloride, i.e., both the CSCMC-B1 and CS-CMC-CUR-B1 were irregular to rod shaped, but CS-CMC-B2, CS-CMC-B3, CS-CMCCUR-B2 and CS-CMC-CUR-B3 were spherical and irregular. Size of all the composites was $1-5 \mu \mathrm{m}$; some of the particles were even smaller than $1 \mu \mathrm{m}$ (Fig. 3). Surface morphology of microparticles chelated with TPP, i.e., CS-CMC-S1 showed that there were approximately uniform spheres (Fig. 4a), and others CS-CMC-S2, CS-CMC-S3 were found to be irregular to rod shaped (Fig. 4b, c). SEM analysis of CS-CURCMC-S1, CS-CUR-CMC-S2, and CS-CUR-CMC-S3 showed that they were fluffy in appearance, and smooth, irregular in shape (Fig. 4b, d, f). TPP concentration might have influenced the shape variations. Martins et al. (2012) found that CS/TPP molar ratio at $\mathrm{pH} 5$ favored the formation of more compacted particles, whereas CS/TPP molar ratio at $\mathrm{pH} 2$ did not favor the formation of small particles as well as those formed were irregularly shaped large clusters. Therefore, the $\mathrm{CS} / \mathrm{TPP}$ ratio and the $\mathrm{pH}$ clearly influenced the size and porosity of CS/TPP particles. They also found the CS/ TPP particles to have irregular shapes. 
Fig. 12 Antibacterial activity of CUR on Pseudomonas aeruginosa using acetic acid as solvent. a chelated with $0.2 \%$ $\mathrm{BaCl}_{2}$ and loaded with drug (CS-CMC-CUR-B1),

b chelated with $0.4 \% \mathrm{BaCl}_{2}$ and loaded with drug (CSCMC-CUR-B2), c chelated with $0.6 \% \mathrm{BaCl}_{2}$ and loaded with drug (CS-CMC-CUR-

B3). d chelated with $0.2 \%$ TPP and loaded with drug (CSCMC-CUR-S1), e chelated with $0.4 \%$ TPP and loaded with drug (CS-CMC-CUR-S2), f chelated with $0.6 \%$ TPP and loaded with drug (CS-CMCCUR-S3)
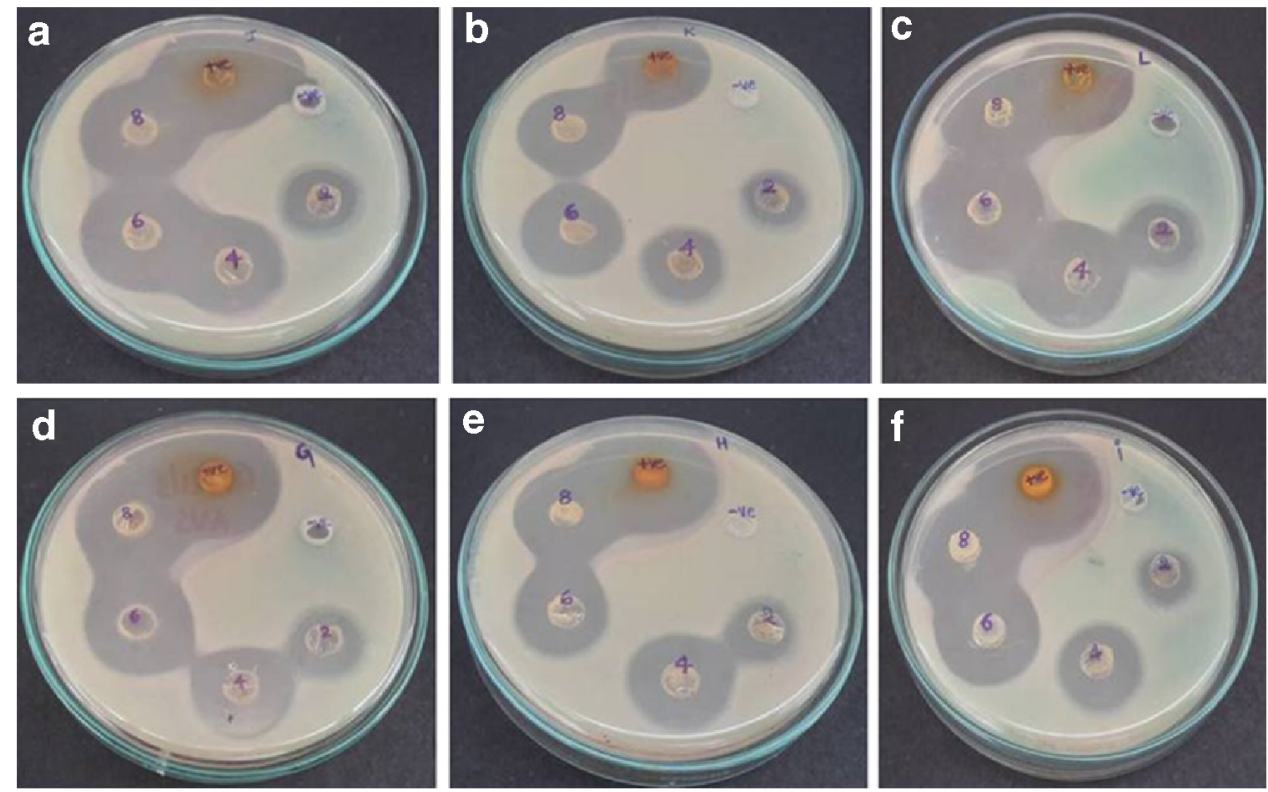

Table 1 Antibacterial activity of CUR against Pseudomonas aeruginosa using water as a solvent

\begin{tabular}{|c|c|c|c|c|c|c|}
\hline \multirow[t]{2}{*}{ Type of microparticles } & \multirow[t]{2}{*}{ Positive control } & \multirow[t]{2}{*}{ Negative control } & \multicolumn{4}{|c|}{ Zone of inhibition at various Concentration $(\mathrm{cm})$} \\
\hline & & & $20 \mu \mathrm{l}$ & $40 \mu 1$ & $60 \mu 1$ & $80 \mu 1$ \\
\hline CS-CUR-CMC-B1 & $-\mathrm{ve}$ & $-\mathrm{ve}$ & $-\mathrm{ve}$ & $-\mathrm{ve}$ & $-\mathrm{ve}$ & $-\mathrm{ve}$ \\
\hline CS-CUR-CMC-B2 & $-\mathrm{ve}$ & $-\mathrm{ve}$ & $-\mathrm{ve}$ & $-\mathrm{ve}$ & $-\mathrm{ve}$ & $-\mathrm{ve}$ \\
\hline CS-CUR-CMC-B3 & $-\mathrm{ve}$ & $-\mathrm{ve}$ & $-\mathrm{ve}$ & $-\mathrm{ve}$ & $-\mathrm{ve}$ & $-\mathrm{ve}$ \\
\hline CS-CUR-CMC-S1 & $-\mathrm{ve}$ & $-\mathrm{ve}$ & $-\mathrm{ve}$ & $-\mathrm{ve}$ & $-\mathrm{ve}$ & $-\mathrm{ve}$ \\
\hline $\mathrm{CS}-\mathrm{CUR}-\mathrm{CMC}-\mathrm{S} 2$ & $-\mathrm{ve}$ & $-\mathrm{ve}$ & $-\mathrm{ve}$ & $-\mathrm{ve}$ & $-\mathrm{ve}$ & $-\mathrm{ve}$ \\
\hline CS-CUR-CMC-S3 & - ve & $-\mathrm{ve}$ & $-\mathrm{ve}$ & $-\mathrm{ve}$ & $-\mathrm{ve}$ & $-\mathrm{ve}$ \\
\hline
\end{tabular}

-ve negative

Table 2 Antibacterial activity of CUR against Pseudomonas aeruginosa using ethanol as solvent

\begin{tabular}{|c|c|c|c|c|c|c|}
\hline \multirow[t]{2}{*}{ Type of microparticles } & \multirow[t]{2}{*}{ Positive control } & \multirow[t]{2}{*}{ Negative control } & \multicolumn{4}{|c|}{ Zone of inhibition at various Concentration $(\mathrm{cm})$} \\
\hline & & & $20 \mu \mathrm{l}$ & $40 \mu 1$ & $60 \mu 1$ & $80 \mu 1$ \\
\hline CS-CUR-CMC-B1 & $-\mathrm{ve}$ & $-\mathrm{ve}$ & $-\mathrm{ve}$ & $-\mathrm{ve}$ & $-\mathrm{ve}$ & $-\mathrm{ve}$ \\
\hline CS-CUR-CMC-B2 & - ve & $-\mathrm{ve}$ & $-\mathrm{ve}$ & $-\mathrm{ve}$ & $-\mathrm{ve}$ & $-\mathrm{ve}$ \\
\hline CS-CUR-CMC-B3 & $-\mathrm{ve}$ & $-\mathrm{ve}$ & $-\mathrm{ve}$ & $-\mathrm{ve}$ & $-\mathrm{ve}$ & $-\mathrm{ve}$ \\
\hline CS-CUR-CMC-S1 & $-\mathrm{ve}$ & $-\mathrm{ve}$ & $-\mathrm{ve}$ & $-\mathrm{ve}$ & $-\mathrm{ve}$ & $-\mathrm{ve}$ \\
\hline CS-CUR-CMC-S2 & - ve & $-\mathrm{ve}$ & $-\mathrm{ve}$ & $-\mathrm{ve}$ & $-\mathrm{ve}$ & $-\mathrm{ve}$ \\
\hline CS-CUR-CMC-S3 & $-\mathrm{ve}$ & $-\mathrm{ve}$ & $-\mathrm{ve}$ & $-\mathrm{ve}$ & $-\mathrm{ve}$ & $-\mathrm{ve}$ \\
\hline
\end{tabular}

-ve negative

\section{Encapsulation efficiency}

From Figs. 5 and 6, the encapsulation of CUR in all the composites was increasing with increase in time. CUR, a hydrophobic drug encapsulation and stabilization is influenced by cross-linking agents. CS-CMC-S2 and CS-
CMC-S3 microcarriers showed a faster encapsulation than others. The initial concentration of CUR also plays an important role in the encapsulation efficiency of carriers. TPP cross-linked with CS-CMC carriers have shown a higher encapsulation efficiency than other barium chloride cross-linked carriers, because particles are cleaved 
Table 3 Antibacterial activity of CUR against Pseudomonas aeruginosa using phosphate buffer solution as solvent

\begin{tabular}{|c|c|c|c|c|c|c|}
\hline \multirow[t]{2}{*}{ Type of microparticles } & \multirow[t]{2}{*}{ Positive control } & \multirow[t]{2}{*}{ Negative control } & \multicolumn{4}{|c|}{ Zone of inhibition at various concentration $(\mathrm{cm})$} \\
\hline & & & $20 \mu \mathrm{l}$ & $40 \mu \mathrm{l}$ & $60 \mu \mathrm{l}$ & $80 \mu 1$ \\
\hline CS-CUR-CMC-B1 & 1.6 & $-\mathrm{ve}$ & $-\mathrm{ve}$ & $-\mathrm{ve}$ & $-\mathrm{ve}$ & 1.3 \\
\hline CS-CUR-CMC-B2 & 1.2 & - ve & $-\mathrm{ve}$ & $-\mathrm{ve}$ & $-\mathrm{ve}$ & 0.9 \\
\hline CS-CUR-CMC-B3 & 1.6 & $-\mathrm{ve}$ & $-\mathrm{ve}$ & $-\mathrm{ve}$ & $-\mathrm{ve}$ & $-\mathrm{ve}$ \\
\hline CS-CUR-CMC-S1 & 1.4 & $-\mathrm{ve}$ & $-\mathrm{ve}$ & $-\mathrm{ve}$ & $-\mathrm{ve}$ & $-\mathrm{ve}$ \\
\hline CS-CUR-CMC-S2 & 1.6 & $-\mathrm{ve}$ & $-\mathrm{ve}$ & $-\mathrm{ve}$ & 0.9 & 1.1 \\
\hline CS-CUR-CMC-S3 & 1.6 & $-\mathrm{ve}$ & $-\mathrm{ve}$ & $-\mathrm{ve}$ & 1 & 1.2 \\
\hline
\end{tabular}

-ve negative

Table 4 Antibacterial activity of CUR against Pseudomonas aeruginosa using acetic acid as solvent

\begin{tabular}{|c|c|c|c|c|c|c|}
\hline \multirow[t]{2}{*}{ Type of microparticles } & \multirow[t]{2}{*}{ Positive control } & \multirow[t]{2}{*}{ Negative control } & \multicolumn{4}{|c|}{ Zone of inhibition at various concentration $(\mathrm{cm})$} \\
\hline & & & $20 \mu \mathrm{l}$ & $40 \mu 1$ & $60 \mu 1$ & $80 \mu 1$ \\
\hline CS-CUR-CMC-B1 & 3.5 & $-\mathrm{ve}$ & 2.2 & 2.5 & 2.8 & 3 \\
\hline CS-CUR-CMC-B2 & 2.9 & $-\mathrm{ve}$ & 1.5 & 1.8 & 2.2 & 2.5 \\
\hline CS-CUR-CMC-B3 & 3.5 & $-\mathrm{ve}$ & 1.8 & 2.5 & 3 & 3.2 \\
\hline CS-CUR-CMC-S1 & 3.5 & $-\mathrm{ve}$ & 2 & 2.5 & 2.7 & 3 \\
\hline CS-CUR-CMC-S2 & 3.2 & $-\mathrm{ve}$ & 1.2 & 2.2 & 2.1 & 2.8 \\
\hline CS-CUR-CMC-S3 & 3.5 & $-\mathrm{ve}$ & 1.3 & 2.2 & 2.6 & 2.7 \\
\hline
\end{tabular}

-ve negative

regularly, and without aggregation, when CUR enter into carriers, it easily catches and binds. It is reported that encapsulation efficiency of the nanoparticles was affected by increasing the TPP concentration (Zhang et al. 2010), but in this study, good encapsulation was found with higher concentration of TPP.

\section{In vitro drug release kinetics}

Drug release kinetics of drug-epitomized microcarriers was studied at pH 6.8 in phosphate buffer by dialysis membrane technique. The release kinetics of barium chloride is different for each concentration. In the plot of CS-CURCMC-B1, higher amount of drug is released in $30 \mathrm{~min}$ and steeps down at $90 \mathrm{~min}$ and gradual rise in release of drug reaches at $180 \mathrm{~min}$. In the plot of CS-CUR-CMC-B2, more amount of drug is released at $60 \mathrm{~min}$, and drug release plateaued till $180 \mathrm{~min}$. CS-CUR-CMC-B3 has steady release of drug till $120 \mathrm{~min}$, and more amount of drug is released at $150 \mathrm{~min}$ (Fig. 7). The plots of CSCUR-CMC-S1 and CS-CUR-CMC-S2 showed similar kind of drug release kinetics. More amount of drug is released in first $20 \mathrm{~min}$, and it steeps down in next $40 \mathrm{~min}$, and the graph reaches steady state till $180 \mathrm{~min}$, and that of CS-CUR-CMC-S3 shows steady release in drug till $120 \mathrm{~min}$ and there was a sharp peak at $150 \mathrm{~min}$ which reveals that there is more amount of drug released at
$150 \mathrm{~min}$ (Fig. 8). Overall, drug release kinetic study reveals that $\mathrm{CS}-\mathrm{CUR}-\mathrm{CMC}-\mathrm{B} 3$ holds drug for more time and gradually releases it. Gradually, CUR released from the carrier, $25 \%$ of CUR release took more than $70 \mathrm{~min}$, and then, 25-60\% of CUR released within $15 \mathrm{~min}$. This is due to the hydration of composites and diffuses, creates voids by swelling of the polymers, and more number of positively charged amines present in CS, there is less possibility of penetration of acidic media and, thereby, gradual release of CUR from CS-CMC carriers (Mukhopadhyay et al. 2014). Bisht et al. (2007) found nanoparticle-encapsulated formulation of CUR - nanocurcumin-utilizing the micellar aggregates of cross-linked and random copolymers of $N$-isopropylacrylamide (NIPAAM), with $N$-vinyl-2-pyrrolidone (VP) and poly(ethyleneglycol)monoacrylate (PEG-A) to disperse in aqueous media than free CUR (Figs. 9, 10, 11, 12).

\section{In vitro controlled release studies}

No antibacterial activity was observed when water and ethanol were used as solvent in case of microcarriers chelated with TPP and barium chloride (Tables 1,2). This is due to the inability of the solvent to dissolve the CS microcomposites. When phosphate buffer solution ( $\mathrm{pH} 6$ ) was used as a solvent, zones of inhibition of 1.3 and $1.2 \mathrm{~cm}$ were found in $\mathrm{CS}$ CUR-CMC-B1 and CS-CUR-CMC-S3 composites, 
respectively; it means that $\mathrm{pH} 6$ favors the release of $\mathrm{CS}$ microcomposites (Table 3). Even acetic acid showed the maximum zone of inhibition in all the microcomposites (Table 4); the reason must be that it dissolves the CS better than the other solvents used and favors the better release of the drug encapsulated. Buzia et al. (2015) found CS TPP microspheres loaded with vancomycin to be effective against Streptococcus pyogenes and Streptococcus faecalis and also suggested it for oral delivery of vancomycin.

\section{Conclusion}

Microcarriers were synthesized utilizing biopolymers and chelating agents, and the produced microcarriers were epitomized with CUR. Microcarriers with and without encapsulated CUR were characterized using SEM and FTIR. SEM analysis studies showed that barium chloride surface morphology is likely smooth rod to spherical in shape (size of 1-10 $\mu \mathrm{m}$ ) and that of TPP was found to be irregular to spheres. FTIR shows all the functional groups related to CMC, CS and CUR. The TPP cross-linked with CS-CMC carriers have higher encapsulation efficiency than barium chloride. In drug release kinetics study, it was observed that CS-CUR-CMC-B3 (0.6\% barium chloride) was good at holding the drug for long time and steady release of drug. While studying antimicrobial activity against $P$. aeruginosa, no activity was observed when ethanol and water were taken as a solvent for CUR. When phosphate buffer solution was used as a solvent, the maximum zone of inhibition was observed in CS-CUR-CMC$\mathrm{B} 1$, and the activity was seen only in the highest concentration. Antimicrobial activity showed that all drug-encapsulated microcarriers, when acetic acid was used as solvent, showed activity in all concentrations, and $0.6 \%$ barium chloride showed maximum zone.

\section{Acknowledgments Authors thank Sathyabama University for their support.}

\section{Compliance with ethical standards}

Conflict of interest Authors have no conflict of interest.

Open Access This article is distributed under the terms of the Creative Commons Attribution 4.0 International License (http:// creativecommons.org/licenses/by/4.0/), which permits unrestricted use, distribution, and reproduction in any medium, provided you give appropriate credit to the original author(s) and the source, provide a link to the Creative Commons license, and indicate if changes were made.

\section{References}

Ammon HPT, Wahl MA (1991) Pharmacology of Curcuma longa. Planta Med 57(1):1-7
Andrianov AK, Payne LG (1998) Polymeric carriers for oral uptake of microparticles. Adv Drug Deliv Rev 34:155-170

Benetton SA, Kedor-Hackmann ERM, Santoro MIRM, Borges VM (1998) Visible spectrophotometric and first-derivative UV spectrophotometric determination of rifampicin and isoniazid in pharmaceutical preparations. Talanta 47(3):639-643

Bigucci F, Abruzzo A, Vitali B, Saladini B, Cerchiara T, Gallucci MC et al (2015) Vaginal inserts based on chitosan and carboxymethylcellulose complexes for local delivery of chlorhexidine: preparation, characterization and antimicrobial activity. Int J Pharm 478:456-463

Bisht S, Feldmann G, Soni S, Ravi R, Karikar C, Maitra A, Maitra A (2007) Polymeric nanoparticle-encapsulated curcumin ("nanocurcumin"): a novel strategy for human cancer therapy. J Nanobiotechnol 5:3

Buzia OD, Dima C, Dima S (2015) Preparation and characterization of chitosan microspheres for vancomycin delivery. Farmacia 63(6):897-902

Calvo P, Remunan-Lopez C, Vila-Jato JL, Alonso MJ (1997) Novel hydrophilic chitosan-polyethylene oxide nanoparticles as protein carriers. J Appl Polym Sci 63:125-132

Cerchiara T, Abruzzo A, Parolin C, Vitali B, Bigucci F, Gallucci MC, Nicoletta FP, Luppi B (2016) Microparticles based on chitosan/carboxymethylcellulose polyelectrolyte complexes for colon delivery of vancomycin. Carbohydr Polym 143:124-130

Chandy T, Sharma CP (1990) Chitosan-as a biomaterial biomater. Artif Cells Artif Organs 18:1-24

Cruickshank R (1962) Mackie and Mc Cartney's handbook of bacteriology: a guide to the laboratory diagnosis and control of infection, 10th ed. E and S Livingstone Limited, Edinburgh, London

Dima C, Cotarlet M, Alexe P, Dima S (2014) Microencapsulation of essential oil of pimento [Pimenta dioica (L) Merr.] by chitosan/ k-carrageenan complex coacervation method. Innov Food Sci Emerg Technol 20:203-211

Edwards DA, Caponetti JG, Mintzes J, Hrkach J, Lotan N, Langer R (1997) Large porous particles for pulmonary drug delivery. Science 276(5320):1868-1871

Garcia A, Leonardi D, Piccirilli NG, Mamprin ME, Olivieri AC, Lamas MC (2015) Spray drying formulation of albendazole microspheres by experimental design. In vitro-in vivo studies. Drug Dev Ind Pharm 41:244-252

Hua S (2014) Comparison of in vitro dialysis release methods of loperamide-encapsulated liposomal gel for topical drug delivery. Int J Nanomed 9:735-744

Jisha JP, Thulasidasan AKT, Anto RJ, Devika NC, Ashwanikumar N, Vinodkumar GS (2015) Curcumin entrapped folic acid conjugated PLGA-PEG nanoparticles exhibit enhanced anticancer activity by site specific delivery. RSC Adv 5(25):25518-25524

Kas HS (1997) Chitosan: properties, preparations and application to microparticulate systems. J Microencapsul 14(6):689-711

Kurita K (1986) Chemical modification of chitin and chitosan. In: Muzzarelli RA, Jeuniaux C, Gooday GW (eds) Chitin in nature and technology. Plenum, New York, pp 287-293

Liu CH, Huang HY (2012) Antimicrobial activity of curcumin-loaded myristic acid microemulsions against Staphylococcus epidermidis. Chem Pharm Bull (Tokyo) 60(9):1118-1124

Lu S, Liu M, Ni B (2010) An injectable oxidized carboxymethylcellulose $N$-succinyl-chitosan hydrogel system for protein delivery. Chem Eng J 160:779-787

Martins AF, de Oliveira DM, Pereira AGB, Rubira AF, Muniz EC (2012) Chitosan/TPP microparticles obtained by microemulsion method applied in controlled release of heparin. Int J Biol Macromol 51(5):1127-1133

Mi FL, Shyu SS, Lee ST, Wong TB (1999) Kinetic study of chitosantripolyphosphate complex reaction and acid-resistive properties 
of the chitosan-tripolyphosphate gel beads prepared by in-liquid curing method. J Polym Sci B 37:1551-1564

Mitra A, Dey B (2011) Chitosan microspheres in novel drug delivery systems. Indian J Pharm Sci 73(4):355-366

Mukerjee A, Vishwanatha JK (2009) Formulation, characterization and evaluation of curcumin-loaded PLGA nanospheres for cancer therapy. Anticancer Res 29(10):3867-3875

Mukhopadhyay P, Sarkar K, Bhattacharya S, Mishra R, Kundu PP (2014) Efficient oral insulin delivery by dendronized chitosan: in vitro and in vivo studies. RSC Adv 4(83):43890-43902

Niamsa N, Sittiwet C (2009) Antimicrobial activity of Curcuma longa aqueous extract. J Pharmacol Toxicol 4(4):173-177

Okada H (1997) One- and three-month release injectable microspheres of the LH-RH superagonist leuprorelin acetate. Adv Drug Deliv Rev 28(1):43-70

Parize AL, Stulzer HK, Laranjeira MCM, Brighente MDC, de-Souza TCR (2012) Evaluation of chitosan microparticles containing curcumin and crosslinked with sodium tripolyphosphate produced by spray drying. Quím Nova 35(6):1127-1132

Sanford PA (1989) Chitosan: commercial uses and potential applications. In: Skjak G, Anthonsen T, Sanford P (eds) Chitin and chitosan-sources, chemistry, biochemistry, physical properties and applications. Elsevier, London, pp 51-69

Shahidi F, Synowiecki J (1991) Isolation and characterization of nutrients and value-added products from snow crab (Chionoecetes opilio) and shrimp (Pandalus borealis) processing discards. J Agric Food Chem 39(8):1527-1532

Smart JD, Kellaway ID, Worthington HEC (1994) An in vitro investigation of mucosa-adhesive materials for use in controlled drug delivery. J Pharm Pharmacol 36(5):295-299
Takishima J, Onishi H, Machida Y (2002) Prolonged intestinal absorption of cephradine with chitosan-coated ethylcellulose microparticles in rats. Biol Pharm Bull 25:1498-1502

Wang Q, Jamal A, Detamore MS, Berkland S (2011) PLGA-chitosan/ PLGA-alginate nanoparticle blends as biodegradable colloidal gels for seeding human umbilical cord mesenchymal stem cells. J Biomed Mater Res A 96(3):520-527

Wright LE, Frye JB, Gorti B, Timmermann BN, Funk JL (2013) Bioactivity of turmeric-derived curcuminoids and related metabolites in breast cancer. Curr Pharm Des 19(34):6218-6225

Yao KD, Peng T, Yin YJ, Xu MX (1995) Microcapsules/microspheres related to chitosan. J Macromol Sci 35:155-180

Yu H, Huang Q (2012) Improving the oral bioavailability of curcumin using novel organogel-based nanoemulsions. J Agric Food Chem 60(21):5373-5379

Yue Z, You Z, Yang Q, Lv P, Yue H, Wang B, Ni D, Wei W, Ma G (2013) Molecular structure matters: PEG-b-PLA nanoparticles with hydrophilicity and deformability demonstrate their advantages for high-performance delivery of anti-cancer drugs. J Mater Chem B 1:3239-3247

Zhang HL, Wu SH, Tao Y, Zang LQ, Su ZQ (2010) Preparation and characterization of water-soluble chitosan nanoparticles as protein delivery system. J Nanomater 2010:1

Zhao LM, Shi LE, Zhang ZL, Chen JM, Shi DD, Yang J, Tang ZX (2011) Preparation and application of chitosan nanoparticles and nanofibers. Braz J Chem Eng 28(3):353-362

Zimmer A, Kreuter J (1995) Microspheres and nanoparticles used in ocular delivery systems. Adv Drug Deliv Rev 16:61-73 